\title{
BIBLIOGRAPHY OF ACADEMICIAN GLIGOR JOVANOVSKI
}

\section{Papers concerning metal saccharinates and their complexes with $N$-donor ligands}

[1] Г. Јовановски, А. Николовски, Б. Шоптрајанов, Бипиридински адукти на сахаринатите на бакар, никел и кобалт: инфрацрвени спектри, екстракција и примена при спектрофотометриско определување на сахарин, 7. Југ̄o-

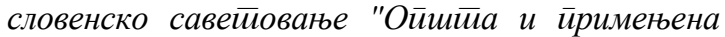
сиеккйроскойија", Ниш, 1978, Зборник, Београд, 133-138 (1980).

G. Jovanovski, A. Nikolovski, B. Šoptrajanov, Bipyridine adducts of saccharinates of copper, nickel and cobalt: Infrared spectra, extraction and application for spectrophotometric determination of saccharin, 7. Yugoslav Symposium "General and Applied Spectroscopy", Niš, 1978, Proceedings, Beograd, 133-138 (1980).

[2] G. Jovanovski, B. Kamenar, Two ionic saccharinates: (1a) Sodium saccharinate $2 / 3$ hydrate, $\mathrm{C}_{7} \mathrm{H}_{4} \mathrm{NO}_{3} \mathrm{SNa} \cdot 2 / 3 \mathrm{H}_{2} \mathrm{O}$; (1b) Magnesium disaccharinate heptahydrate, $\left(\mathrm{C}_{7} \mathrm{H}_{4} \mathrm{NO}_{3} \mathrm{~S}\right)_{2} \mathrm{Mg} \cdot 7 \mathrm{H}_{2} \mathrm{O}$, Cryst. Struct. Comm. 11, 247-255 (1982).

[3] B. Kamenar, G. Jovanovski, Manganese(II) saccharinate hexahydrate, $\mathrm{Mn}\left(\mathrm{C}_{7} \mathrm{H}_{4} \mathrm{NO}_{3} \mathrm{~S}\right)_{2} \cdot 6 \mathrm{H}_{2} \mathrm{O}$, and isomorphism with the analogous $\mathrm{Fe}, \mathrm{Co}, \mathrm{Ni}, \mathrm{Zn}$ and Cd complexes, Cryst. Struct. Comm. 11, 257261 (1982).

[4] B. Kamenar, G. Jovanovski, D. Grdenić, Mercury(II) saccharinate, $\mathrm{Hg}\left(\mathrm{C}_{7} \mathrm{H}_{4} \mathrm{NO}_{3} \mathrm{~S}\right)_{2}$, Cryst. Struct. Comm. 11, 263-268 (1982).

[5] G. Jovanovski, B. Šoptrajanov, Spectra-structure correlations in the isomorphous series of metal(II) saccharinates hexahydrates, J. Mol. Struct. 143, 159-162 (1986).

[6] G. Jovanovski, B. Kamenar, Structural study of some metal saccharinates, 5. Italian-Yugoslav Crystallographic Congress, Padova, Proceedings, C12 (4 pages) (1986).

[7] G. Ferguson, G. Jovanovski, B. Kaitner, B. Kamenar, Crystal structure of chloromercury(II) saccharinate, $\mathrm{C}_{7} \mathrm{H}_{4} \mathrm{ClHgNO}_{3} \mathrm{~S}$, 5. Italian-Yugoslav Crystallographic Congress, Padova, Proceedings, C49 (3 pages) (1986).

[8] A. Hergold-Brundić, G. Jovanovski, B. Kamenar, Crystal structure of lead(II) saccharinate monohydrate, $\mathrm{Pb}\left(\mathrm{C}_{7} \mathrm{H}_{4} \mathrm{NO}_{3} \mathrm{~S}\right)_{2} \cdot \mathrm{H}_{2} \mathrm{O}$, 5. Italian-Yugoslav Crystallographic Congress, Padova, Proceedings, C51 (2 pages) (1986).
[9] O. Grupče, G. Jovanovski, Polymorphism in thiosaccharin, Vestn. Slov. Kem. Druš. 33, 53-54 (1986).

[10] Г. Јовановски, Б. Андоновиќ, О. Групче, Препарација и испитување на изоморфизмот кај тиосахаринатите на калциум, стронциум и бариум,

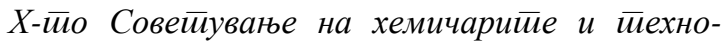

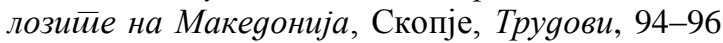
(1987).

G. Jovanovski, B. Andonović, O. Grupče, Preparation and investigation of isomorphysm in thiosaccharinates of calcium, strontium and barium, $X$ Symposium of Chemists and Technologists of Macedonia, Skopje, Proceedings, 94-96, (1987).

[11] G. Jovanovski, A. Hergold-Brundić, B. Kamenar, Structure of lead(II) disaccharinate monohydrate, Acta Crystallogr. C44, 63-66 (1988).

[12] G. Jovanovski, B. Kamenar, G. Ferguson, B. Kaitner, Structure of chloromercury(II) saccharinate, Acta Crystallogr. C44, 616-618 (1988).

[13] G. Jovanovski, B. Šoptrajanov, Bonding of the carbonyl group in metal saccharinates: Correlation with the infrared spectra, J. Mol. Struct. 174, 467472 (1988).

[14] A. Hergold-Brundić, B. Kamenar, G. Jovanovski, Structure of the 1:1 complex of mercury(II) saccharinate with bipyridyl, Acta Crystallogr. C45, 556-558 (1989).

[15] G. Jovanovski, O. Grupče, B. Šoptrajanov, The O$\mathrm{H}$ and $\mathrm{O}-\mathrm{D}$ stretching vibrations in the hydrates of sodium and potassium saccharinate: Spectrastructure correlations, J. Mol. Struct. 219, 61-66 (1990).

[16] M. Penavić, G. Jovanovski, O. Grupče, Crystal structure of sodium thiosaccharinate monohydrate, Acta Crystallogr. C46, 2341-2344 (1990).

[17] G. Jovanovski, B. Šoptrajanov, B. Kamenar, Spectra-structure correlations in some metal saccharinates, Bull. Chem. Technol. Macedonia, 8, 47-66 (1990).

[18] M. Penavić, O. Grupče, G. Jovanovski, Crystal structure of potassium thiosaccharinate monohydrate, Acta Crystallogr. C47, 1821-1823 (1991).

[19] A. Hergold-Brundić, O. Grupče, G. Jovanovski, Structure of bis(2,2'-bipyridyl)(saccharinato-N)copper(II) saccharinate dihydrate, Acta Crystallogr. C47, 2659-2660 (1991).

[20] E. Kleinpeter, D. Strohl, G. Jovanovski, B. Šoptrajanov, Metal-to-ligand bonding in some 
metal saccharinates: A ${ }^{13} \mathrm{C}$ NMR study, J. Mol. Struct. 246, 185-188 (1991).

[21] O. Grupče, G. Jovanovski, B. Šoptrajanov, Monohydrates of the thiosaccharinates of sodium and potassium: Spectra-structure correlations, J. Mol. Struct. 267, 197-202 (1992).

[22] S. Tančeva, G. Jovanovski. B. Šoptrajanov, Infrared spectra of protiated and deuterated lead(II) saccharinate monohydrate: Spectra-structure correlations, Spectrosc. Lett. 25(7), 927-941 (1992).

[23] O. Grupče, G. Jovanovski, B. Šptrajanov, The N$\mathrm{H}, \mathrm{N}-\mathrm{D}$ and $\mathrm{C}=\mathrm{S}$ stretching regions in the infrared spectrum of thiosaccharin: Comparison with the spectrum of saccharin, J. Mol. Struct. 293, 113116 (1993).

[24] S. Tančeva, G. Jovanovski, B. Šoptrajanov, Infrared spectrum of silver saccharinate: Structural inferences, Bull. Chem. Technol. Macedonia, 12, 11-15 (1993).

[25] O. Grupče, G. Jovanovski, V. Mirčeski, Spectrastructure correlations in 2,2'-bipyridine mercury(II) saccharinate: Comparison with mercury(II) saccharinate and chloromercury(II) saccharinate, Spectrosc. Lett. 27, 691-699 (1994).

[26] O. Grupče, M. Penavić, G. Jovanovski, Structural study of thiosaccharin by single crystal X-ray diffraction and infrared spectroscopy, J. Chem. Crystallogr. 24, 581-586 (1994).

[27] G. Jovanovski, S. Tančeva, B. Šoptrajanov, The $\mathrm{SO}_{2}$ stretching vibrations in some metal saccharinates: Spectra-structure correlations, Spectrosc. Lett. 28, 1095-1109 (1995).

[28] G. Jovanovski, D. Spasov, S. Tančeva, B. Šoptrajanov, Structural characteristics of the hydrates of the saccharinates of calcium, strontium and barium, Acta Chim. Slov. 43, 41-50 (1996).

[29] O. Grupče, G. Jovanovski, V. Mirčeski, Structural characteristics of 2,2'-bipyridine saccharinato complexes with cobalt(II), nickel(II) and zinc(II), Bull. Chem. Technol. Macedonia, 15, 87-92 (1996).

[30] O. Grupče, G. Jovanovski, Infrared spectra of protiated and deuterated lead(II) 2,2'-bipyridine saccharinato monohydrate, J. Mol. Struct. 408/409, 333-336 (1997).

[31] Lj. Pejov, G. Jovanovski, O. Grupče, B. Šoptrajanov, The Influence of inherent pseudo JahnTeller instability on the stretching vibrations of water molecules in the isomorphous metal(II) saccharinate hexahydrates, J. Mol. Struct. 410-411, 365-369 (1997).

[32] Lj. Pejov, G. Jovanovski, O. Grupče, B. Šoptrajanov, Infrared investigation of $\left[\mathrm{Cu}(\mathrm{sac})_{2}\left(\mathrm{H}_{2} \mathrm{O}\right)_{4}\right]$ $\times 2 \mathrm{H}_{2} \mathrm{O}$ - a pseudo Jahn-Teller complex, Acta Chim. Slovenica, 44, 197-211 (1997).

[33] O. Grupče, G. Jovanovski, Preparation and characterization of mercury(II) thiosaccharinate. Analysis of mercury-to-thiosaccharin bonding character by comparison with mercury(II) saccharinate and mercury(II) chlorosaccharinate, Anal. Labor. 7, 912 (1998).

[34] O. Grupče, G. Jovanovski, B. Šoptrajanov, A. Hergold-Brundić, Structure of bis(2,2'-bipyridyl)(saccharinato- $N$ )copper(II) saccharinate dihydrate. Addendum. Acta Crystallogr. C54, 890-891 (1998).

[35] G. Jovanovski, P. Naumov, O. Grupče, B. Kaitner, Structural study of monoaquabis(pyridine)bis(saccharinato)copper(II), $\left[\mathrm{Cu}\left(\mathrm{H}_{2} \mathrm{O}\right)(\text { py })_{2}(\mathrm{sac})_{2}\right]$, Eur. J. Sol. St. Inorg. Chem. 35, 231-242 (1998).

[36] G. Jovanovski, P. Naumov, O. Grupče, B. Kaitner, Tetraaquabis(pyridine)metal(II) saccharinate tetrahydrate, $\left[\mathrm{M}\left(\mathrm{H}_{2} \mathrm{O}\right)_{4}(\mathrm{py})_{2}\right](\mathrm{sac})_{2} \times 4 \mathrm{H}_{2} \mathrm{O} ; \mathrm{M}=\mathrm{Co}, \mathrm{Ni}$, Eur. J. Sol. St. Inorg. Chem. 35, 579-590 (1998).

[37] P. Naumov, G. Jovanovski, O. Grupče, Fourier transform infrared study of monoaquabis(pyridine)bis(saccharinato)copper(II): Spectra-structure correlations, J. Mol. Struct. 482-483, 121-124 (1999).

[38] Lj. Pejov, G. Jovanovski, O. Grupče, B. Šoptrajanov, Anharmonicity of water stretching vibrations in isomorphous metal(II) saccharinates hexahydrates, $J$. Mol. Struct. 482-483, 115-120 (1999).

[39] P. Naumov, G. Jovanovski, FT Infrared spectra of binuclear copper(II) imidazole saccharinato complex: Correlation with the structure, Spectrosc. Lett. 32, 237-256 (1999).

[40] O. Grupče, G. Jovanovski, B. Kaitner, P. Naumov, Structural investigation of $\mathrm{di}-\mu$-chloro-bis[saccharinatopyridinemercury(II)] by X-ray diffraction and FT IR spectroscopy, Croat. Chim. Acta, 72, 465-476 (1999).

[41] G. Jovanovski, A. Hergold-Brundić, O. Grupče, D. Matković-Čalogović, Structure of (2,2'-bipyridyne)lead(II) saccharinate monohydrate, J. Chem. Crystallogr. 29, 233-237 (1999).

[42] P. Naumov, G. Jovanovski, Infrared study of the binuclear cadmium(II) imidazole saccharinato complex: Comparison with the copper(II) compound, Acta Chim. Slov. 46, 389-404 (1999).

[43] P. Naumov, G. Jovanovski, V. Jordanovska, B. Boyanov, The Thermal decomposition of the pyridine saccharinates of $\mathrm{Co}, \mathrm{Ni}$ and $\mathrm{Cu}$ : A correlation of the structural and the infrared data, J. Serb. Chem. Soc. 64, 609-620 (1999).

[44] P. Naumov, G. Jovanovski, Synthesis and comparative vibrational study of two novel cesium saccharinates: Spectroscopic evidence for a saccharin adduct, $16^{\text {th }}$ Congress of Chemists and Technologists of Macedonia, Skopje, Book of Papers, 2730 (1999).

[45] P. Naumov, G. Jovanovski, Vibrational Studies of the Imidazole and Pyridine Adducts of Metal(II) Saccharinates. I. The OH/OD and NH/ND Stretching Regions of the Cobalt(II) and Nickel(II) Complexes, 
$16^{\text {th }}$ Congress of Chemists and Technologists of Macedonia, Skopje, Book of Papers, 63-66 (1999).

[46] P. Naumov, G. Jovanovski, S. Abbrent, L.-E. Tergenius, Thermal Behavior of the Saccharinates of $\mathrm{K}, \mathrm{Na}, \mathrm{Rb}, \mathrm{Cs}$ and $\mathrm{NH}_{4}, 16^{\text {th }}$ Congress of Chemists and Technologists of Macedonia, Skopje, Book of Papers, 67-70 (1999).

[47] P. Naumov, O. Grupče, G. Jovanovski, Raman Spectrum of the Binuclear Copper(II) Imidazole Saccharinato Complex, $16^{\text {th }}$ Congress of Chemists and Technologists of Macedonia, Skopje, Book of Papers, 71-74 (1999).

[48] O. Grupče, G. Jovanovski, B. Kaitner, Structural Study of Cadmium Complexes with 2,2' 'bipyridine and Saccharin, $16^{\text {th }}$ Congress of Chemists and Technologists of Macedonia, Skopje, Book of Papers, 55-58 (1999).

[49] G. Jovanovski, Metal saccharinates and their complexes with $\mathrm{N}$-donor ligands, Croat. Chem. Acta, 73, 843-868 (2000).

[50] P. Naumov, G. Jovanovski, On the geometry of the saccharinato ligand/ion in the metal saccharinates: Crystallographic survey and theoretical study, Struct. Chem. 11, 19-33 (2000).

[51] P. Naumov, G. Jovanovski, Vibrational study and spectra-structure correlations in ammonium saccharinate: Comparison with the alkali saccharinates, Spectrochim. Acta A, 56, 1305-1318 (2000).

[52] P. Naumov, O. Grupče, G. Jovanovski, Experimental and theoretical Raman study of the binuclear copper(II) imidazole saccharinato complex, $J$. Raman Spectrosc. 31, 475-479 (2000).

[53] P. Naumov, G. Jovanovski, Vibrational studies of the solid imidazole and pyridine aducts of metal(II) saccharinates. I. The OH/OD and NH/(ND) stretching regions of the cobalt(II) and nickel(II) complexes, Sol. St. Scien. 2, 249-256 (2000).

[54] A. Ćahil, G. Jovanovski, O. Grupče, Isomorphism in the thiosaccharinates of $\mathrm{Mg}, \mathrm{Co}(\mathrm{II}), \mathrm{NI}(\mathrm{II})$ and $\mathrm{Zn}$ (II): Comparison with the analogous metal saccharinates, Bull. Chem. Technol. Macedonia, 19, 9-16 (2000).

[55] P. Naumov, G. Jovanovski, S. Abbrent, L.-E. Tergenius, Thermal behaviour of the saccharinates of $\mathrm{K}^{+}$, $\mathrm{Na}^{+}, \mathrm{Rb}^{+}, \mathrm{Cs}^{+}$and $\mathrm{NH}_{4}^{+}$: Structural inferences, Thermochim. Acta, 359, 123-130 (2000).

[56] P. Naumov, G. Jovanovski, Vibrational study of two novel cesium saccharinates. Spectroscopic evidence for organic molecule embeded in ionic salt, Vib. Spectrosc. 24 201-211 (2000).

[57] P. Naumov, G. Jovanovski, On the coordination in metal saccharinates, J. Coord. Chem. 54, 63-79 (2001).

[58] P. Naumov, G. Jovanovski, Outer-sphere coordination, $\mathrm{N}$-coordination and $\mathrm{O}$-coordination of the deprotonated saccharin in copper(II) saccharinato complexes. Implications for the saccharinato carbonyl stretching frequency, Inorg. Chim. Acta, 314, 154-162 (2001).

[59] P. Naumov, G. Jovanovski, Spectra-structure correlations in solid metal saccharinates. I. The carbonyl stretchings, J. Mol. Struct. 563-564, 335339 (2001).

[60] P. Naumov, G. Jovanovski, A. Todorovska, Vibrational studies of the solid imidazole and pyridine adducts of metal(II) saccharinates. II. Mn(II) and Fe(II) imidazole saccharinates, J. Mol. Struct. 563-564, 341-345 (2001).

[61] P. Naumov, V. Jordanovska, O. Grupče, B. Boyanov, G. Jovanovski, Thermal behaviour of the $\mathrm{N}$-donor adducts of metal saccharinates. I. 2,2'bipyridine saccharinato complexes of $\mathrm{Co}(\mathrm{II})$, $\mathrm{Ni}(\mathrm{II}), \mathrm{Cu}(\mathrm{II}), \mathrm{Zn}(\mathrm{II})$ and $\mathrm{Pb}(\mathrm{II})$, J. Therm. Anal. Cal. 65, 59-67 (2001).

[62] P. Naumov, V. Jordanovska, O. Grupče, B. Boyanov, G. Jovanovski, Thermal behaviour of the $\mathrm{N}$-donor adducts of metal saccharinates. II. 1,10phenantroline saccharinato complexes of $\mathrm{Co}(\mathrm{II})$, $\mathrm{Ni}(\mathrm{II}), \mathrm{Cu}(\mathrm{II}), \mathrm{Zn}(\mathrm{II})$ and $\mathrm{Pb}(\mathrm{II}), J$. Therm. Anal. Cal. 65, 871-880 (2001).

[63] P. Naumov, G. Jovanovski, An update to the combined vibrational-diffraction experimental and theoretical studies of small biologically important cyclic imides: Reference to saccharin, Curr. Org. Chem. 5, 1059-1077 (2001).

[64] P. Naumov, V. Jordanovska, B. Boyanov, G. Jovanovski, Thermal behaviour of the $\mathrm{N}$-donor adducts of metal saccharinates. III. Imidazole saccharinates of $\mathrm{Co}(\mathrm{II}), \mathrm{Ni}(\mathrm{II})$ and $\mathrm{Cd}(\mathrm{II})$, J. Therm. Anal. Cal. 66, 469-477 (2001).

[65] P. Naumov, G. Jovanovski, S.-Z. Hu, I.-H. Suh, I. A. Razak, S. Chantrapromma, H.-K. Fun, S. W. Ng, On the short carbonyl bond in bis[ $\mu$-1,2-benzisothiazol$3(2 H)$-one1,1-dioxido- $\left.k^{2} N: O\right]\{$ bis[1,2-benzisothiazol-3(2H)-one1,1-dioxido- $k N]$-bis(imidazole)copper(II) \}, Acta Crystallogr. C57, 1016-1019 (2001).

[66] O. Grupče, G. Jovanovski, B. Kaitner, P. Naumov, Monoaquabis(2,2'-bipyridine)saccharinatozinc(II) saccharinate. I. Structural study by single crystal X-ray diffraction, FT IR spectroscopy and DS calorimetry, J. Struct. Chem. 42, 926-934 (2001).

[67] S. Cakir, P. Naumov, I. Bulut, E. Bicer, O. Cakir, G. Jovanovski, I. A. Razak, S. Chatrapromma, H.K. Fun, S. W. Ng, Diaquabis(nicotinamide)bis $(o-$ sulfobenzimidato- $N$ )cadmium(II), Acta Crystallogr. E57, m431-m432 (2001).

[68] P. Naumov, G. Jovanovski, J. V. Hanna, I. A. Razak, S. Chantrapromma, H.-K. Fun, S. W. Ng, Diaquabis(4,4'-bipyridine)copper(II) $\operatorname{di}(o$-sulfobenzimidate) dichloromethane solvate, a two-dimensional $\mathrm{Cu}_{4}\left(4,4^{\prime}-\mathrm{C}_{5} \mathrm{H}_{4} \mathrm{NC}_{5} \mathrm{H}_{4} \mathrm{~N}\right)_{4}$ rhombic grid clathrating guest dichloromethane, Inorg. Chem. Commun. 4, 766-768 (2001). 
[69] P. Naumov, G. Jovanovski, A. Todorovska, Vibrational studies of the solid imidazole and pyridine adducts of metal(II) saccharinates. III. $\mathrm{Zn}$ and $\mathrm{Hg}(\mathrm{II})$ imidazole saccharinates, Spectrochim. Acta A, 58, 1903-1910 (2002).

[70] P. Naumov, G. Jovanovski, Y. Ohashi, Spectrastructure correlations in solid saccharinates. II. $A b$ initio molecular structures and vibrational spectra of $N$-substituted saccharins at the HF Level, Sol. St. Scien. 4, 271-283 (2002).

[71] P. Naumov, G. Jovanovski, S. W. Ng, Aquabis(2,4'bipyridine)di( $o$-sulfobenzimidato)copper(II) at $95 \mathrm{~K}$, Acta. Crystallogr. E58, m16-m17 (2002).

[72] H. Icbudak, P. Naumov, M. Ristova G. Jovanovski, Structural studies of bis $(o$-sulfobenzimidato)praseodymium(III) chloride hexahydrate, J. Mol. Struct. 606, 77-86 (2002).

[73] P. Naumov, S. Cakir, I. Bulut, E. Bicer, O. Cakir, G. Jovanovski, A. R. Ibrahim, A. Usman, H.-K. Fun, S. Chantrapromma, S. W. Ng, Crystal structure of di(saccharinato-O)lead(II)nicotinamide hemihydrate, Main Group. Metal Chem. 25, 175-176 (2002).

[74] P. Naumov, G. Jovanovski, M. Ristova, I. A. Razak, S. Cakir, S. Chantrapromma, H.-K. Fun, S. $\mathrm{W} . \mathrm{Ng}$, Coordination of deprotonated saccharin in copper(II) complexes. Structural role of the saccharinate directed by the ancillary $\mathrm{N}$-heterocyclic ligands, Z. Anorg. Allg. Chem. 628, 2930-2939 (2002).

[75] G. Jovanovski, O. Grupče, Vibrational study and spectra-structure correlations in mercury(II) chlorosaccharinate, Bull. Chem. Technol. Maced, 21, 117-124 (2002).

[76] H. Icbudak, H. Olmez, O. K. Yesilil, F. Arslan, P. Naumov, G. Jovanovski, A. R. Ibrahim, A. Usman, H.-K. Fun, S. Chantrapromma, S. W. Ng, Syntheses, characterization and crystal structure of a novel amine adducts of metal saccharinates, orotates and salicilates, J. Mol. Struct. 657, 255-270 (2003).

[77] G. Jovanovski, B. Kaitner, O. Grupče, P. Naumov, Crystal structure, infrared and Raman spectra of tripotassium trisaccharinate dihydrate, $\mathrm{K}_{3}\left(\mathrm{C}_{7} \mathrm{H}_{4} \mathrm{NO}_{3} \mathrm{~S}\right)_{3} \times 2 \mathrm{H}_{2} \mathrm{O}$, Cent. Eur. J. Chem. 2, 254-275 (2004).

[78] P. Naumov, G. Jovanovski, O. Grupče, B. Kaitner, D. A. Rae, S. W. Ng, Solid-state structure and temperature/evacuation-induced dehydration of sodium saccharinate 1.875 hydrate, Angew. Chem. Int. Ed. 44, 1251-1254 (2005).

[79] G. Jovanovski, A. Ćahil, O. Grupče, Lj. Pejov, Vibrational analysis of thiosaccharin and thiosaccharinate anion. A gradient-corrected density functional and experimental study, J. Mol. Struct. 784, 7-17 (2006).
[80] P. Naumov, G. Jovanovski, S. Tančeva, S. W. Ng, Crystal structure and spectroscopic characterization of lithium saccharinate 11/6 hydrate, hygroscopic and potentially physiologically active compound, $Z$. Anorg. Allg. Chem. 632, 454-460 (2006).

[81] P. Naumov, G. Jovanovski, K. Sakurai, Thermally induced saccharinate ligand flips in single crystals of bis(imidazole)bis(saccharinato)copper(II) close to ambient temperature, Crys. Grow. Des. 6, 815817 (2006).

[82] G. Jovanovski, A. Ćahil, O. Grupče, Infrared spectra of thiosaccharinates of cadmium and lead. Comparison with the analogous metal saccharinates, Contributions, Sec. Math. Tech. Sci., MANU, 27/28, 15-27 (2006/2007).

[83] Lj. Pejov, G. Jovanovski, O. Grupče, B. Šoptrajanov, Anharmonicity of water stretching vibrations in series of isomorphous crystalline hydrates. Copper and manganese saccharinates hexahydrates, Spectrochem. Acta A, 66, 419-426 (2007).

[84] P. Naumov, Lj. Pejov, G. Jovanovski, T. Stafilov, M. Taseska, E. Stojanovska, Unilateral exclusion of Jahn-Teller-inactive $d^{5} \quad \mathrm{Mn}\left(\mathrm{H}_{2} \mathrm{O}\right)_{4}\left(\mathrm{C}_{7} \mathrm{H}_{4} \mathrm{NO}_{3} \mathrm{~S}\right)_{2}{ }^{2+}$ guests by strongly distorted host $d^{9}$ $\mathrm{Cu}\left(\mathrm{H}_{2} \mathrm{O}\right)_{4}\left(\mathrm{C}_{7} \mathrm{H}_{4} \mathrm{NO}_{3} \mathrm{~S}\right)_{2}{ }^{2+}$ lattice, Crys. Grow. Des., 8, 1319-1326 (2008).

[85] G. Jovanovski, P. Makreski, B. Šoptrajanov, Vibrational study and spectra-structure correlations in magnesium disaccharinate heptahydrate, $\mathrm{Mg}(\mathrm{sac})_{2} \cdot 7 \mathrm{H}_{2} \mathrm{O}$, Maced. J. Chem. Chem. Eng., 27, 1-8 (2008).

[86] F. Quentel, K. Stankoska, O. Grupče, G. Jovanovski, V. Mirčeski, Electrochemistry of saccharinate anion at liquid interfaces, Electrochem. Commun., 13, 1476-1478 (2011).

[87] G. Jovanovski, Crystallography in Macedonia Collaboration of Macedonian and Croatian Crystallographers. Scientific Conference "Crystallography in Croatia", on the occasion of the 20th anniversary of the Croatian Crystallographic Association, Zagreb, Book of Papers, 43-53, (2013).

\section{Papers concerning minerals from the Republic of Macedonia}

[88] M. Trajkovska, B. Šoptrajanov, G. Jovanovski, T. Stafilov, Vibrational Spectra of some sulfide minerals from Alšar, J. Mol. Struct., 267, 191-196 (1992).

[89] B. Šoptrajanov, M. Trajkovska, I. Gržetić, G. Jovanovski, T. Stafilov, Infrared spectra of $\mathrm{M}_{3}^{\mathrm{I}} \mathrm{M}^{\mathrm{III}} \mathrm{S}_{3}$ type synthetic minerals $\left(\mathrm{M}^{\mathrm{I}}=\mathrm{Tl}\right.$ or $\mathrm{Ag}, \mathrm{M}^{\mathrm{III}}=\mathrm{As}$ or Sb), N. Jb. Miner. Abh., 166/1, 83-89 (1993).

[90] M. Trajkovska, B, Šoptrajanov, T. Stafilov, G. Jovanovski, Determination of lorandite and realgar in mineral mixtures using infrared spectroscopy, Geol. Maced., 7, 55-59 (1993). 
[91] B. Šoptrajanov, M. Trajkovska, G. Jovanovski, T. Stafilov, Infrared spectra of lorandite and some other minerals from Allchar, N. Jb. Miner. Abh., 167, 329-337 (1994).

[92] B. Šoptrajanov, M. Trajkovska, T. Stafilov, G. Jovanovski, I. Gržetić, Infrared spectra of three $\mathrm{M}^{\mathrm{I}} \mathrm{M}^{\mathrm{III}} \mathrm{S}_{2}$ type synthetic minerals $\left(\mathrm{M}^{\mathrm{I}}=\mathrm{Ag}\right.$ or $\mathrm{Tl}$, $\mathrm{M}^{\mathrm{III}}=\mathrm{Sb}$ or As), Spectrosc. Lett., 30, 79-87 (1997).

[93] G. Jovanovski, V. Stefov, B. Jovanovski, B. Šoptrajanov, B. Kaitner, Minerals from Macedonia: I. Analytical application of powder X-ray diffraction patterns of calcite and aragonite, 16th Congress of Chemists and Technologists of Macedonia, Skopje, Book of Papers, 43-46, (1999).

[94] В. Стефов, С. Димитровска, Г. Јовановски, Б. Шоптрајанов, Минерали од Македонија: II. Вибрациони спектри на некои сулфатни минерали, 16. Конг̄рес на хемичарийе и

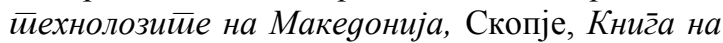
йpygови, 47-50, (1999).

V. Stefov, S. Dimitrovska, G. Jovanovski, B. Šoptrajanov, Minerals from Macedonia: II. Vibrational spectra of some sulfate minerals, $16^{\text {th }}$ Congress of Chemists and Technologists of Macedonia, Skopje, Book of Papers, 47-50, (1999).

[95] Б. Шоптрајанов, В. Стефов, И. Кузмановски, Г. Јовановски, Фурие трансформни инфрацрвени спектри на некои фосфатни минерали, 16.

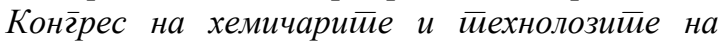
Макеgонија, Скопје, Книг̄a на йруgови, 103106, (1999).

B. Šoptrajanov, V. Stefov, I. Kuzmanovski, G. Jovanovski, Fourier-transform infrared spectra of some phosphate minerals, $16^{\text {th }}$ Congress of Chemists and Technologists of Macedonia, Skopje, Book of Papers, 103-106, (1999).

[96] G. Jovanovski, V. Stefov, B. Jovanovski, B. Šoptrajanov, B. Boev, Minerals from Macedonia. III. Determination of calcite and aragonite in mineral mixtures using FT IR spectroscopy, Geol. Maced., 13, 69-74 (1999).

[97] V. Stefov, G. Jovanovski, B. Šoptrajanov, B. Minčeva-Šukarova, S. Dimitrovska, B. Boev, Minerals from Macedonia. V. Characterization of gypsum, barite and their synthetic analogues by FTIR and Raman spectroscopy, Geol. Maced., 14, 61-66 (2000).

[98] P. Makreski, G. Jovanovski, V. Stefov, B. Minčeva-Šukarova, B. Kaitner, B. Boev, Minerals from Macedonia. VI. Separation and identification of some sulfide minerals, Geol. Maced., 15-16, 43-50 (2001-2002).

[99] G. Jovanovski, B. Minčeva Šukarova, P. Makreski, B. Šoptrajanov, W. P. Griffith, R. L. Willis, Minerals from Macedonia: VII. Raman spectra of sulfide minerals from the Allchar deposite (stibnite, realgar, orpiment and lorandite), Eighteenth Inter- national Conference of Raman Spectroscopy, Proceedings, 931-932, Budapest (2002).

[100] G. Jovanovski, B. Minčeva Šukarova, P. Makreski, V. Stefov, W. P. Griffith, R. L. Willis, Minerals from Macedonia: VIII. Raman spectra of some sulfide minerals from the localities in Eastern Macedonia, Eighteenth International Conference of Raman Spectroscopy, Proceedings, 933-934, Budapest (2002).

[101] B. Boev, V. Bermanec, T. Serafimovski, S. Lepitkova, S. Mikulčić, M. Šoufek, G. Jovanovski, T. Stafilov, M. Najdoski, Allchar mineral assemblage, Geol. Maced., 15-16, 1-23 (2001-2002).

[102] G. Jovanovski, V. Stefov, B. Šoptrajanov, B. Boev, Minerals from Macedonia. IV. Discrimination between some carbonate minerals by FTIR spectroscopy, N. Jh. Miner. Abh., 177, 241-253 (2002).

[103] P. Makreski, V. Zajkova Paneva, G. Jovanovski. T. Stafilov, D. Zendelovska, AAS and AES-ICP determination of trace elements in some iron minerals, $3^{\text {rd }}$ Aegean Analytical Chemistry Days, Proceedings, 440-443, Polihnitos, Lesbos (2002).

[104] B. Minceva-Sukarova, G. Jovanovski, P. Makreski, B. Soptrajanov, W. Griffith, R. Willis, I. Grzetic, Vibrational spectra of $\mathrm{M}^{\mathrm{I}} \mathrm{M}^{\mathrm{III}} \mathrm{S}_{2}$ type synthetic minerals $\left(\mathrm{M}^{\mathrm{I}}=\mathrm{Tl}\right.$ or $\mathrm{Ag}$ and $\mathrm{M}^{\mathrm{III}}=\mathrm{As}$ or Sb), J. Mol. Struct., 651-653, 181-189 (2003).

[105] P. Makreski, G. Jovanovski, Minerals from Macedonia. IX. Distinction between some rhombohedral carbonates by FT IR spectroscopy, Bull. Chem. Technol. Maced., 22, 25-32 (2003).

[106] G. Jovanovski, B. Boev, P. Makreski, M. Najdoski, G. Mladenovski, Minerals from Macedonia. XI. Silicate varieties and their localities - identification by FT IR spectroscopy, Bull. Chem. Technol. Maced., 22, 111-141 (2003).

[107] P. Makreski, G. Jovanovski, B. MinčevaŠukarova, B. Šoptrajanov, A. Green, B. Engelen, I. Grzetić, Vibrational spectra of $\mathrm{M}_{3}^{\mathrm{I}} \mathrm{M}^{\mathrm{III}} \mathrm{S}_{3}$ type synthetic minerals $\left(\mathrm{M}^{\mathrm{I}}=\mathrm{Tl}\right.$ or $\mathrm{Ag}$ and $\mathrm{M}^{\mathrm{III}}=\mathrm{As}$ or Sb), Vib. Spectrosc., 35, 59-66 (2004).

[108] P. Makreski, G. Jovanovski, B. Kaitner, T. Stafilov, B. Boev, D. Cibrev, Minerals from Macedonia. X. Separation and identification of some oxide minerals by FT IR spectroscopy, AAS, EAS-ICP and powder XRD, N. Jh. Miner. Abh., 180, 215243 (2004).

[109] P. Makreski, G. Jovanovski, T. Stafilov, B. Boev, Minerals from Macedonia. XII. The dependance of quartz and opal color on trace element composition - AAS, FT IR and micro-Raman spectroscopy study, Bull. Chem. Technol. Maced., 23, 171-184 (2004).

[110] P. Makreski, G. Jovanovski, S. Stojančeska, Minerals from Macedonia. XIII. Vibrational spectra of some commonly appearing nesosilicate minerals, J. Mol. Struct., 744-747, 79-92 (2005). 
[111] P. Makreski, G. Jovanovski, S. Dimitrovska, Minerals from Macedonia. XIV. Identification of some sulfate minerals by vibrational (infrared and Raman) spectroscopy, Vib. Spectrosc., 39, 229-239 (2005).

[112] M. Taseska T. Stafilov, P. Makreski, G. Jovanovski, Determination of trace elements in some copper minerals by atomic absorption spectrometry, Ovidius University Annals of Chemistry, 16, 43-46 (2005).

[113] B. Boev, G. Jovanovski, P. Makreski, V. Bermanec, Minerals from Macedonia. XV. Sivec mineral assemblage, Geol. Maced., 19, 39-56 (2005).

[114] R. Jaćimović, P. Makreski, V. Stibilj, T. Stafilov, G. Jovanovski, Characterization of some iron minerals from Republic of Macedonia using instrumental neutron activation analysis, Geol. Maced., 19, 33-38 (2005).

[115] G. Jovanovski, P. Makreski, B. Šoptrajanov, B. Kaitner, B. Boev, Minerals from Macedonia. Complementary use of vibrational spectroscopy and powder X-ray diffraction for identification and detection purposes, Contributions, Sec. Math. Tech. Sci. MANU, XXVI, 7-84 (2005).

[116] P. Makreski, G. Jovanovski, A. Gajović, Minerals from Macedonia. XVII. Vibrational spectra of common appearing amphibole minerals, Vib. Spectrosc., 40, 98-109 (2006).

[117] P. Makreski, G. Jovanovski, A. Gajović, T. Biljan, D. Angelkovski, R. Jaćimović, Minerals from Macedonia. XVI. Vibrational spectra of some common appearing pyroxene and pyroxenoids, $J$. Mol. Struct., 788, 102-114 (2006).

[118] M. Taseska T. Stafilov, P. Makreski, R. Jaćimović, V. Stibilj, G. Jovanovski, Liquid-liquid extraction and determination of trace elements in iron minerals by atomic absorption spectrometry, Geol. Maced., 20, 33-38 (2006).

[119] P. Makreski, G. Jovanovski, B. Kaitner, A. Gajović, T. Biljan, Minerals from Macedonia. XVIII. Vibrational spectra of some sorosilicates, Vib. Spectrosc., 44, 162-170 (2007).

[120] V. Šontevska, G. Jovanovski, P. Makreski, Minerals from Macedonia. XIX. Vibrational spectroscopy as identificational tool for some sheet silicate minerals, J. Mol. Struct., 834-836, 318-327 (2007).

[121] P. Naumov, P. Makreski, G. Jovanovski, Direct atomic-scale observation of linkage isomerization of $\mathrm{As}_{4} \mathrm{~S}_{4}$ clusters during the photoinduced transition of realgar to pararealgar, Inorg. Chem., 46, 10624-10631 (2007).

[122]R. Jaćimović, M. Taseska, V. Stibilj, P. Makreski, T. Stafilov, G. Jovanovski, Element composition of some copper minerals from the Republic of Macedonia, Geol. Maced., 21, 87-91 (2007).

[123] P. Makreski, G. Jovanovski, Minerals from Macedonia. XXII. Laser-induced fluorescence bands in the FT-Raman spectrum of almandine mineral, $J$. Raman Spectrosc., 39, 1210-1213 (2008).

[124] V. Šontevska, G. Jovanovski, P. Makreski, A. Raškovska, B. Šoptrajanov, Minerals from Macedonia. XXI. Vibrational spectroscopy as identificational tool for some phyllosilicate minerals, Acta Chim. Slov., 55, 757-766 (2008).

[125] M. Taseska, P. Makreski, V. Stibilj, R. Jaćimović, T. Stafilov, G. Jovanovski, Determination of trace elements in chalcopyrite $\left(\mathrm{CuFeS}_{2}\right)$ by $k_{0}$-instrumental neutron activation analysis after matrix elements removal, Macedonian J. Chem. Chem. Eng., 27, 141-147 (2008).

[126] P. Makreski, R. Jaćimović, V. Stibilj, T. Stafilov, G. Jovanovski, The elemental composition of some iron minerals from Macedonia using neutron activation analysis, Radiochim. Acta, 96, 855-861 (2008).

[127] Г. Јовановски, Атоми, молекули, кристали и

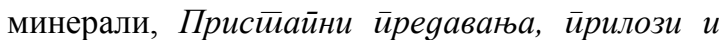
библиог̄рафија на новитее членови на Макеgон-

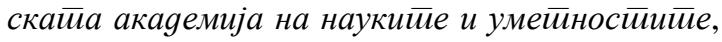
XVI, 57-113 (2008).

G. Jovanovski, Atoms, molecules, crystals and minerals, Opening Addresses, Contributions and Bibliography of the new members of the Macedonian Academy of Sciences and Arts, XVI, 57-113 (2008).

[128] P. Makreski, G. Jovanovski, Minerals from Macedonia. XXIII. Spectroscopic and structural characterization of schorl and beryl cyclosilicates, Spectrochim. Acta A, 73, 460-467 (2009).

[129] G. Jovanovski, P. Makreski, B. Kaitner, B. Boev, Silicate minerals from Macedonia. Complementary use of vibrational spectroscopy and X-ray powder diffraction for identification and detection purposes, Croat. Chem. Acta, 82, 363-386 (2009). Erratum, Croat. Chem. Acta, 85, CCXI-CCXIII (2012).

[130] B. Boev, G. Jovanovski, P. Makreski, Minerals from Macedonia. XX. Geological setting, lithological rock types and identification of the minerals from Ržanovo Fe-Ni deposit, Turkish J. Earth Sci., 18, 631-652 (2009).

[131] P. Makreski, G. Jovanovski, B. Kaitner, Minerals from Macedonia. XXIV. Spectra-structure characterization of tectosilicates, J. Mol. Struct., 924 926, 413-419 (2009).

[132] G. Jovanovski, P. Makreski, B. Kaitner, B. Šoptrajanov, Minerals from Macedonia. X-ray powder diffraction $v s$. vibrational spectroscopy in mineral identification, Contributions, Sec. Math. Tech. Sci., MANU, XXX, 1-2, 7-34 (2009).

[133] M. Tasevska, R. Jaćimović, V. Stibilj, T. Stafilov, P. Makreski, G. Jovanovski, Is copper removal by electrolysis from copper minerals appropriate 
method for determination of trace elements?, $\mathrm{Nucl}$. Instr. Meth. Phys. Res., A, 622, 449-452 (2010).

[134] P. Naumov, P. Makreski, Gj. Petruševski, T. Runčevski, G. Jovanovski, Visualization of a discrete solid-state process with steady-state X-ray diffraction: observation of hopping of sulfur atoms in single crystals of realgar, J. Am. Chem. Soc., 132, 11398-11401 (2010).

[135] P. Makreski, T. Runčevski, G. Jovanovski, Minerals from Macedonia. XXVI. Characterization and spectra-structure correlations for grossular and uvarovite. Raman study supported by IR spectroscopy, J. Raman Spectrosc., 42, 72-77 (2011).

[136] N. Stamatovska, P. Makreski, Lj. Pejov, G. Jovanovski, Minerals from Macedonia. XXVII. Theoretical and experimental study of the vibrational spectra of endemic nežilovite, J. Mol. Struct., 993, 104-108 (2011).

[137]P. Makreski, G. Jovanovski, T. Runčevski, R. Jaćimović, Simple and efficient method for detection of traces of rare earth elements in minerals by Raman spectroscopy, Maced. J. Chem. Chem. Eng., 30, 241-250 (2011).

[138] G. Jovanovski, B. Boev, P. Makreski, Minerals from the Republic of Macedonia with an introduction to mineralogy, Українсько-Макеgонський науковий збірник, 5, 305-326, Київ (2011).

[139] M. Taseska, R. Jaćimović, V. Stibilj, T. Stafilov, P. Makreski, G. Jovanovski, Determination of trace elements in some minerals by $k_{0}$-instrumental neutron activation analysis, Appl. Rad. Isot., 70, 35-39 (2012).

[140] B. Boev, G. Jovanovski, P. Makreski, Geology and mineralogy of Allchar Sb-As-Ti-Au Deposit, Geol. Maced., 3, 215-232 (2012).

[141] P. Makreski, S. Jovanovski, Lj. Pejov, G. Kloess, H.J. Hoebler, G. Jovanovski, Theoretical and experimental study of the vibrational spectra of sarkinite, $\mathrm{Mn}_{2}\left(\mathrm{AsO}_{4}\right)(\mathrm{OH})$ and adamite, $\mathrm{Zn}_{2}\left(\mathrm{AsO}_{4}\right)(\mathrm{OH})$, Spectrochim. Acta A, 113, 37-42 (2013).

[142] P. Makreski, G. Petruševski, S. Ugarković, G. Jovanovski, Laser-induced transformation of stibnite $\left(\mathrm{Sb}_{2} \mathrm{~S}_{3}\right)$ and other structurally related salts, Vib. Spectrosc., 68, 177-182 (2013).

[143] P. Makreski, G. Jovanovski, B. Boev, MicroRaman spectra of extremely rare and endemic Tlsulfosalts from Allchar deposit, J. Raman spectrosc., 45, 610-617 (2014).

[144] Z. Bujňáková, P. Baláž, P. Makreski, G. Jovanovski, M. Čaplovičová, L. Čaplovič, O. Shpotyuk, A. Ingram, T-C. Lee, J-J. Cheng, J. Sedlák, E. Turianicová and A. Zorkovská, Arsenic sulfide nanoparticles prepared by milling: Properties, free-volume characterization and anti-cancer effects, J. Mater. Sci., 50, 1973-1985 (2015).

[145] P. Makreski, S. Stefov, Lj. Pejov, G. Jovanovski, Theoretical and experimental study of the vibrational spectra of (para)symplesite, $\mathrm{Fe}_{3}\left(\mathrm{AsO}_{4}\right)_{2} \cdot 8 \mathrm{H}_{2} \mathrm{O}$ and hörnesite, $\mathrm{Mg}_{3}\left(\mathrm{AsO}_{4}\right)_{2} \cdot 8 \mathrm{H}_{2} \mathrm{O}$, Spectrochim. Acta, A 144, 155-162 (2015).

[146] P. Makreski, S. Jovanovski, Lj. Pejov, Gj. Petruševski, S. Ugarković, G. Jovanovski, Theoretical and experimental study of the vibrational spectra of liroconite, $\mathrm{Cu}_{2} \mathrm{Al}\left(\mathrm{AsO}_{4}\right)(\mathrm{OH})_{4} \cdot 4 \mathrm{H}_{2} \mathrm{O}$ and bayldonite, $\mathrm{Cu}_{3} \mathrm{~Pb}\left[\mathrm{O}\left(\mathrm{AsO}_{3} \mathrm{OH}\right)_{2}(\mathrm{OH})_{2}\right]$, Vibr. Spectrosc., 79, 36-43 (2015).

[147] T. Runčevski, R.E. Dinnebier, P. Makreski, G. Jovanovski, The Crystal Structure of Symplesite, Z. Anorg. Allg. Chem., 641, (2015). In press.

\section{Other papers}

[148] D. Grdenić, B. Kamenar, B. Korpar-Čolig, M. Sikirica and G. Jovanovski, Tetrakis(trifluoroacetoxymercuri)methane and tetrakis(acetoxymercuri)methane as the reaction products of Hofmann's base with the corresponding acid: X-ray crystallographic evidence, J. Chem. Soc., Chem. Comm. 646-647 (1974).

[149] Б. Шоптрајанов, С. Ѓорѓевиќ, Г. Јовановски, Вибрациони спектри на протонирани, делумно и целосно деутерирани монохидрати на метал(II)калиумови фосфати, 7. Југ̄ословенско

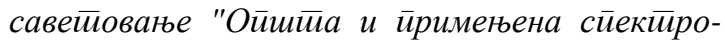
скойија", Ниш, 1978, Зборник, Београд, 119125 (1980).

B. Šoptrajanov, S. Gjorgjević, G. Jovanovski, Vibrational spectra of protonated, partially and totally deuterated monohydrates of metal(II) porassium phosphates, 7. Yugoslav Symposium „General and Applied Spectroscopy”, Niš, 1978, Proceedings, Beograd, 119-125 (1980).

[150] В. Петрушевски, Б. Шоптрајанов, Г. Јовановски, Спектарот на водата кај некои сулфатни и селенатни стипси, 7. Југ̄ословенско савейовање

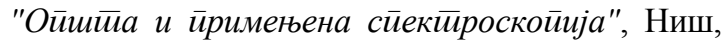
1978, Зборник, Београд, 105-111 (1980).

V. Petruševski, B. Šoptrajanov, G. Jovanovski, The water spectrum in some sulfate and selenate alums, 7. Yugoslav Symposium „General and Applied Spectroscopy", Niš, 1978, Proceedings, Beograd, 105-111 (1980).

[151] М. Фукарова-Јуруковска, Г. Мавродиев, Г. Јовановски, Степен на присутност на метастабилната $\beta$ ' фаза кај калени легури од системот Ag-Zn (27-48 wt. \% Zn) на собна температура, Билиенен на СДФ на Макеgонија, 30-31, 69-73 (1980/81).

M. Fukarova-Jurukovska, G. Mavrodiev, G. Jovanovski, Presence of metastable $\beta$ ' phase in the alloys of the system $\mathrm{Ag}-\mathrm{Zn}$ (27-48 wt. \% Zn) at room temperature, Bull. SDF Macedonia, 30-31, 69-73 (1980/81)

[152] М. Фукарова-Јуруковска, Г. Мавродиев, Г. Јовановски, Прилог кон конкретизирањето на 
границите на $\zeta$-фазата од системот $\mathrm{Ag}-\mathrm{Zn}$ на собна температура, Гog. Зб. Фак. Физ. 31, 121125 (1981).

M. Fukarova-Jurukovska, G. Mavrodiev, G. Jovanovski, Determination of the limits of $\zeta$-phase in the $\mathrm{Ag}-\mathrm{Zn}$ system at room temperature, Annuaire - Physique, 31, 121-125 (1981).

[153] M. Penavić, B. Kamenar, L. Šoptrajanova, G. Jovanovski, B. Šoptrajanov, Preparation, crystal structure and infrared spectra of cis$\left[\mathrm{Pd}\left(\mathrm{NH}_{3}\right)_{2}(\mathrm{CN})_{2}\right]$, God. Jugosl. cent. kristalogr. 17, S59-S61 (1982).

[154] D. Grdenić, B. Kamenar, B. Korpar-Čolig, M. Sikirica, G. Jovanovski, Tetrakis(trifluoroacetoxymercuri)methane, $\mathrm{C}\left(\mathrm{HgOCOCF}_{3}\right)_{4}$, Cryst. Struct. Comm. 11, 565-568 (1982).

[155] L. Šoptrajanova, B. Šoptrajanov, G. Jovanovski, Infrared spectra of cis-[Pd(CN) $\left.)_{2}\left(\mathrm{NH}_{3}\right)_{2}\right], \mathrm{J}$. Mol. Struct. 142, 63-66 (1986).

[156] M. Penavić, L. Šoptrajanova, G. Jovanovski, B. Šoptrajanov, cis-Diamminedicianopalladium(II), Acta Crystallogr., C42, 1283-1284 (1986).

[157] G. Jovanovski, J. Thomas, I. Olovsson, A deformation electron density of potassium oxalate monohydrate at $100 \mathrm{~K}$, Acta Crystallogr. B43, 8592 (1987).

[158] B. Kaitner, G. Jovanovski, I. Janev, Structure of 3,4-dihydroxy-1,6-diphenyl-2,4-hexadiene-1,6-dione(I) and its 1,6-di-p-tolyl analogue (II), Acta Crystallogr. C48, 127-129 (1992).

[159] B. Kaitner, G. Jovanovski, I. Janev, Structure of 2,3-diphenacylquinoxaline, Acta Crystallogr. C48, 129-131 (1992).

[160] M. Cakić, G. Jovanovski, V. Veljković, M. Lazić, M. Stanković, Study on dextran particulation in bottled dextran solutions, Pharmazie, 47, 712-713 (1992).

[161] G. Jovanovski, B. Šoptrajanov, B. Kaitner, L. Prangova, Structural studies of some $o$-substituted S-phenyl thiobenzoates: I. Crystal structure of Sphenyl $o$-chlorothiobenzoate and S-phenyl $o$ bromothiobenzoate, J. Crystallogr. Spectrosc. Res. 23, 49-53 (1993).

[162] B. Šoptrajanov, G. Jovanovski, V. Stefov, I. Kuzmanovski, Vibrational spectra of magnesium hydrogenphosphate trihydrate and of its manganese analogue, Phosphorus, Sulfur and Silicon, 111, 9 (1996).

[163] G. Jovanovski, S. Pocev, B. Kaitner, Crystal structure of magnesium potassium phosphate monohydrate, $\mathrm{MgKPO}_{4} \times \mathrm{H}_{2} \mathrm{O}$, Bull. Chem. Technol. Macedonia, 16, 59-63 (1997).

[164] B. Šoptrajanov, G. Jovanovski, I. Kuzmanovski, V. Stefov, Fourier transform vibrational spectra of magnesium hydrogenphosphate trihydrate. I. The O-H stretching region, Spectroc. Lett. 31, 11911205 (1998).
[165] B. Šoptrajanov, V. Stefov, I. Kuzmanovski, G. Jovanovski, Fourier transform infrared and Raman spectra of manganese hydrogenphosphate trihydrate, J. Mol. Struct. 482-483, 103-107 (1999).

[166] B. Šoptrajanov, V. Stefov, I. Kuzmanovski, G. Jovanovski, Fourier transform vibrational spectra of magnesium hydrogenphosphate trihydrate. II. The 2000-370 $\mathrm{cm}^{-1}$ region, Spectroc. Lett. 32, 703-717 (1999).

[167] G. Jovanovski, B. Šoptrajanov, Lj. Pejov, L. Prangova, Fourier transform infrared and computational study of some o-substituted S-phenyl thiobenzoates, $16^{\text {th }}$ Congress of Chemists and Technologists of Macedonia, Skopje, Book of Papers, 51-54 (1999).

[168] В. Стефов, Б. Шоптрајанов, И. Кузмановски, Г. Јовановски, Фурие трансформни инфрацрвени и рамански спектри на магнезиум хидрогенфосфат трихидрат во областа од 600 до 160 $\mathrm{cm}^{-1}, 16$ Конг̄рес на хемичарийе и иеехнолозийе на Макеgонија, Скопје, Книг̄a на йруgови, 91-94 (1999).

V. Stefov, B. Šoptrajanov, I. Kuzmanovski, G. Jovanovski, Fourier-transform infrared and Raman spectra of magnesium hydrogenphosphate trihydrate in the $600-160 \mathrm{~cm}^{-1}$ region, $16^{\text {th }}$ Congress of Chemists and Technologists of Macedonia, Skopje, Book of Papers, 91-94 (1999).

[169] Lj. Pejov, B. Šoptrajanov, G. Jovanovski, Very low $\mathrm{H}-\mathrm{O}-\mathrm{H}$ bending frequencies. II. Quantum chemical study of the water bending potential in compounds of the $\mathrm{MgKPO}_{4} \cdot \mathrm{H}_{2} \mathrm{O}$ Type, J. Mol. Struct. 563-564, 321-327 (2001).

[170] G. Jovanovski, B. Šoptrajanov, L. Prangova, Lj. Pejov, Ab initio HF SCF and Fourier transform infrared study of the structure and vibrational force field in two $o$-substituted S-phenyl thiobenzoates, Commun. Math. Comp. Chem. 42, 287-296 (2001).

[171] G. Jovanovski, P. Naumov, Chemistry in Macedonia, Bull. Chem. Technol. Maced. 20, 157-182 (2001).

[172] B. Šoptrajanov, G. Jovanovski, Lj. Pejov, Very low $\mathrm{H}-\mathrm{O}-\mathrm{H}$ bending frequencies. III. Fourier transform infrared study of cobalt potassium phosphate monohydrate and manganese potassium phosphate monohydrate, J. Mol. Struct. 613, 4754 (2002).

[173] B. Šoptrajanov, V. Stefov, I. Kuzmanovski, G. Jovanovski, D. Lutz, B. Engelen, Very low H-O$\mathrm{H}$ bending frequencies. IV. Fourier transform infrared spectra of synthetic dittmarite, J. Mol. Struct. 613, 7-14 (2002).

[174] B. Šoptrajanov, Lj. Pejov, G. Jovanovski, V. Stefov, Very low $\mathrm{HOH}$ bending vibrations. V. Quantum chemical study of water bending vibrations in $\mathrm{MgKPO}_{4} \times \mathrm{H}_{2} \mathrm{O}$, J. Mol. Struct. 706, 101106 (2004). 
[175] V. Stefov, A. Hergold-Brundić, B. Šoptrajanov, G. Jovanovski, Tricesium trans-tetraaquadichlorovanadium(III) tetrachloride: Redetermination of the crystal structure and infrared study of the water spectrum, J. Mol. Struct. 707, 109-114 (2004).

[176] G. Petruševski, P. Naumov, G. Jovanovski, S. W. $\mathrm{Ng}$, Unprecedented sodium-oxygen clusters in the solid-state structure of trisodium hydrogentetravalproate monohydrate: A model for the physiological activity of the anticonvulsant drug epilim, Inorg. Chem. Comm., 11, 81-84 (2008).

[177] G. Petruševski, P. Naumov, G. Jovanovski, G. Bogoeva-Gaceva, S. W. Ng, Solid-state forms of sodium valproate, active component of the anticonvulsant drug epilim, ChemMedChem., 3, 13771386 (2008).

[178] P. Naumov, Gj. Petruševski, G. Jovanovski, Vibrational spectra of calcium, strontium and barium valproates. Self-assembly of valproate nanostrands in aqueous solution and in the solid state, CrystEngComm., 12, 2325-2331 (2010).

[179] G. Petruševski, M. Kajdžanoska, S. Ugarkovic, I. Micovski, G. Bogoeva-Gaceva, G. Jovanovski, P. Makreski, Solvatomorphism of codeine phosphate sesquihydrate-Vibrational spectroscopy and thermoanalytical characterization, Vibr. Spectrosc., 63, 460-468 (2012).

[180] Gj. Petruševski, M. Zbačnik, M. Kajdžanoska, S. Ugarković, V. Trimčeski, B. Kaitner, G. Jovanovski, P. Makreski, Pholcodine monohydrate: Crystal structure and polymorphism, J. Cryst. Growth, 375, 119-124 (2013).

[181] Г. Јовановски, Б. Шоптрајанов, В. Стефов, Состојби и перспективи на науката во Република Македонија, Научен собир во Макеgонскайа акаgемија на наукийе и умейносиичйе йо йовоg:

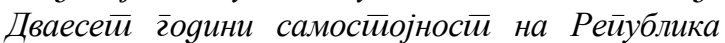
Макеgонија (1991-2011), Скопје, Зборник на

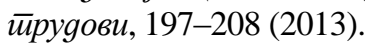

G. Jovanovski, B. Šoptrjanov, V. Stefov, States and perspectives of the science in Macedonia, Symposium at the Macedonian Academy of Sciences and Arts on the Occasion of: The Twenty Years of Independence of the Republic of Macedonia (1991-2011), Skopje, Book of Papers, 197208 (2013).

\section{Professional papers}

[1] Г. Јовановски, 3. Здравковски, Величини, единици и симболи во хемијата, Глас. хем. иеехнол. Макеgонија, 9, 209-222 (1990).

G. Jovanovski, Z. Zdravkovski, Quantities, unites and symbols in chemistry, Bull. Chem. Technol. Macedonia, 9, 209-222 (1990).

[2] Г. Јовановски, 3. Здравковски, 2. Величини, единици и симболи во хемијата, Глас. хем. иеехнол. Макеgонија, 10, 77-81 (1991).
G. Jovanovski, Z. Zdravkovski, 2. Quantities, unites and symbols in chemistry, Bull. Chem. Technol. Macedonia, 10, 77-81 (1991).

[3] Г. Јовановски, Б. Шоптрајанов, 3. Величини, единици и симболи во хемијата, Глас. хем. иеехнол. Макеgонија, 11, 73-78 (1992).

G. Jovanovski, B. Šoptrajanov, 3. Quantities, unites and symbols in chemistry, Bull. Chem. Technol. Macedonia, 11, 73-78 (1992).

[4] Г. Јовановски, Преглед на објавените трудови на научните работници од Институтот за хемија во периодот од 1953-1991 година, Глас. хем. йехнол. Макеgонија, 11, 81-96 (1992).

G. Jovanovski, Survey of the published papers by the Institute of Chemistry staff in the period 19531991, Bull. Chem. Technol. Macedonia, 11, 81-96 (1992).

[5] Г. Јовановски, Б. Шоптрајанов, 4. Величини, единици и симболи во хемијата, Глас. хем. иеехнол. Макеуонија, 12, 51-55 (1993).

G. Jovanovski, B. Šoptrajanov, 4. Quantities, unites and symbols in chemistry, Bull. Chem. Technol. Macedonia, 12, 51-55 (1993).

[6] Г. Јовановски, Б. Шоптрајанов, 5. Величини, единици и симболи во хемијата, Глас. хем. иеехнол. Макеgонија, 13, 47-51 (1994).

G. Jovanovski, B. Šoptrajanov, 5. Quantities, unites and symbols in chemistry, Bull. Chem. Technol. Macedonia, 13, 47-51 (1994).

[7] Г. Јовановски, Двопредметни четиригодишни студии - состојби и преспективи, Месйойо и

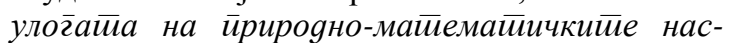

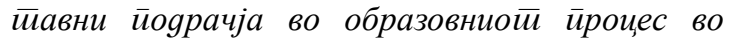

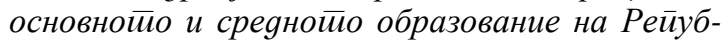
лика Макеgонија, Отешево, Труgови, 167-172 (1994).

G. Jovanovski, Two-subject four-years studies, state and prospectives, Symposium , The role of the natural sciences and mathematics in the educational process of elementary and high-schools in the Republic of Macedonia”, Papers, 167-172 (1994).

[8] Г. Јовановски, Дороти Хоџкин 1910-1994, Глас. хем. ииехнол. Макеуонија, 14, 65-66 (1995).

G. Jovanovski, Doroti Hoxkin 1910-1994, Bull. Chem. Technol. Macedonia, 14, 65-66 (1995).

[9] Г. Јовановски, Б. Шоптрајанов, 6. Величини, единици и симболи во хемијата, Глас. хем. иеехнол. Макеgонија, 14, 117-123 (1995).

G. Jovanovski, B. Šoptrajanov, 6. Quantities, unites and symbols in chemistry, Bull. Chem. Technol. Macedonia, 14, 117-123 (1995).

[10] Г. Јовановски, Кои се нобеловците по хемија, Глас. хем. йехнол. Макеgонија, 16, 161-167 (1997).

G. Jovanovski, Who are the Nobel prize winners in chemistry, Bull. Chem. Technol. Macedonia, 16, 161-167 (1997). 
[11] Г. Јовановски, В. Матевски, Уписната политика

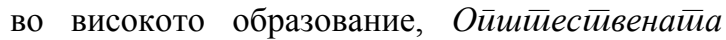

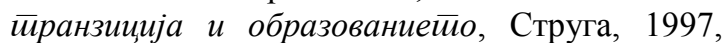
Филозофски факултет, Институт за педагогија, Институт за социологија, Скопје, Книг̄a на

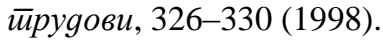

G. Jovanovski, V. Matevski, The enrolement policy in higher education, Symposium ,, Social Transition and Education", Struga, 1997, Faculty of Philosophy, Institute of pedagogy, Institute of Sociology, Skopje, Book of Papers, 326-330 (1998).

[12] V. Urumov, D. Dimoski, G. Jovanovski, M. Karchicka, V. Matevski, The role of the natural sciences in the education process, Phys. Maced. 49, 65-73 (1999).

[13] G. Jovanovski, The Nobel chemistry prize winners through hundred years, Bull. Chem. Technol. Maced. 20, 91-100 (2001).

[14] Н. Гочева, Г. Јовановски, Водата околу нас и во нас, Хемко, 1, 4-7 (2001).

N. Gočeva, G. Jovanovski, The water around us and in our body, Hemko, 1, 4-7 (2001).

[15] G. Jovanovski, The Nobel laureates in chemistry A numeration of Nobelists, Chemical Heritage, 19, 49-51 (2001).

[16] Г. Јовановски, Б. Боев, П. Макрески, Минералите од Македонија, Макеgонско рударсӣво и гееолог̄ија, VIII, 14-17 (2008).

G. Jovanovski, B. Boev, P. Makreski, Minerals from Macedonia, Macedonian Mining and Geology, VIII, 14-17 (2008).

[17] G. Jovanovski, P. Naumov, Crystallography in Macedonia. (In Crystallography in South-Eastern Europe, Part 1), International Union of Crystallography-Newsletter, 19, 19-21 (2011).

[18] Г. Јовановски, Нобелова награда за хемија 2011, Даниел Шехтман, Откривање на квазикристалите, Maced. J. Chem. Chem. Eng., 30, 253-259 (2011).

G. Jovanovski, Nobel Prize in Chemistry - 2011, Daniel Shechtman, Discovery of quasicrystals, Maced. J. Chem. Chem. Eng., 30, 253-259 (2011).

\section{Monographs and books}

[1] G. Ivanovski, G. Jovanovski, O. Popov, V. Petruševski (text and editing), with contributions by D. Dimeski, L. Janićiević, D. Roganović-
Zafirova, Lj. Netkov, Lj. Jordanovski, Faculty of Natural Sciences and Mathematics, Faculty of Natural Sciences and Mathematics, Skopje, 1994.

[2] Б. Шоптрајанов, Г. Јовановски, Л. Андреева, В. Стефов, Физичка хемија I и физичка хемија II, Практикум, Природно-математички факултет, Скопје, 1998 (прво издание); 2003 (второ издание); 2007 (трето издание).

B. Šoptrajanov, G. Jovanovski. L. Andreeva, V. Stefov, Experimental Physical Chemistry I and Physical Chemistry II, Faculty of Natural Sciences and Mathematics, Skopje, 1998 (first edition); 2003 (second edition; 2007 (third edition).

[3] Г. Јовановски, Л. Андреева, Б. Шоптрајанов,

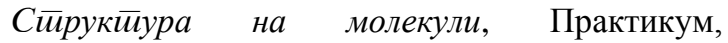
Природно-математички факултет, Скопје, 2001 (прво издание); 2009 (второ издание).

G. Jovanovski, L. Andreeva, B. Šoptrajanov, The Structure of Molecules, Experimental guide, Faculty of Natural Sciences and Mathematics, Skopje, 2001 (first edition); 2009 (second edition).

[4] T. Stafilov, G. Jovanovski (text and editing), Bibliography of the Institute of Chemistry, 19462006, Faculty of Natural Sciences and Mathematics, Skopje, 2006.

[5] Г. Јовановски (главен уредник), К. Богоевски, Б. Вељановски, С. Диневска-Ковкаровска, М. Јоноска, Д. Карчицка, Б. Марковски, Т, Стафилов, В. Чејковска, Н. Шекутковски

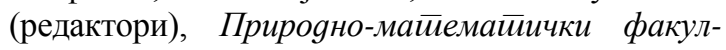
$\bar{u} е \bar{u}$, Скойje, 1946-2006, Природно-математички факултет, Скопје, 2006.

G. Jovanovski (Editor in Chief), K. Bogoevski, B. Veljanovski, S. Dinevska-Ćovkarovska, M. Jonoska, D. Karčicka, B. Markovski, T. Stafilov, V. Čejkovska, N. Šekutkovski (Editors), Faculty of Natural Sciences and Mathematics, Skopje: 19462006, Faculty of Natural Sciences and Mathematics, Skopje, 2006.

[6] T. Stafilov, G. Jovanovski (text and editing), Bibliography of the Institute of Chemistry, 19462011, Faculty of Natural Sciences and Mathematics, Skopje, 2011.

[7] G. Jovanovski, B. Boev, P. Makreski, Minerals from the Republic of Macedonia with an Introduction to Mineralogy. Publisher: Macedonian Academy of Sciences and Arts; co-publisher: Scenpoint, Skopje, 2012. 